\title{
Linking biomechanics and ecology through predator-prey interactions: flight performance of dragonflies and their prey
}

\section{Citation}

Combes, S. A., D. E. Rundle, J. M. Iwasaki, and J. D. Crall. 2012. Linking Biomechanics and Ecology through Predator-Prey Interactions: Flight Performance of Dragonflies and Their Prey. Journal of Experimental Biology 215, no. 6: 903-913. doi:10.1242/jeb.059394.

\section{Published Version}

doi:10.1242/jeb.059394

\section{Permanent link}

http://nrs.harvard.edu/urn-3:HUL.InstRepos:29362007

\section{Terms of Use}

This article was downloaded from Harvard University's DASH repository, and is made available under the terms and conditions applicable to Other Posted Material, as set forth at http:// nrs.harvard.edu/urn-3:HUL.InstRepos:dash.current.terms-of-use\#LAA

\section{Share Your Story}

The Harvard community has made this article openly available.

Please share how this access benefits you. Submit a story.

\section{Accessibility}




\title{
Linking biomechanics and ecology through predator-prey interactions: flight performance of dragonflies and their prey
}

\author{
S. A. Combes*, D. E. Rundle, J. M. Iwasaki and J. D. Crall \\ Harvard University, Concord Field Station, 100 Old Causeway Road, Bedford, MA 01730, USA \\ *Author for correspondence (scombes@oeb.harvard.edu)
}

Accepted 7 November 2011

\begin{abstract}
Summary
Aerial predation is a highly complex, three-dimensional flight behavior that affects the individual fitness and population dynamics of both predator and prey. Most studies of predation adopt either an ecological approach in which capture or survival rates are quantified, or a biomechanical approach in which the physical interaction is studied in detail. In the present study, we show that combining these two approaches provides insight into the interaction between hunting dragonflies (Libellula cyanea) and their prey (Drosophila melanogaster) that neither type of study can provide on its own. We performed $>\mathbf{2 5 0 0}$ predation trials on nine dragonflies housed in an outdoor artificial habitat to identify sources of variability in capture success, and analyzed simultaneous predator-prey flight kinematics from $\mathbf{5 0}$ high-speed videos. The ecological approach revealed that capture success is affected by light intensity in some individuals but that prey density explains most of the variability in success rate. The biomechanical approach revealed that fruit flies rarely respond to approaching dragonflies with evasive maneuvers, and are rarely successful when they do. However, flies perform random turns during flight, whose characteristics differ between individuals, and these routine, erratic turns are responsible for more failed predation attempts than evasive maneuvers. By combining the two approaches, we were able to determine that the flies pursued by dragonflies when prey density is low fly more erratically, and that dragonflies are less successful at capturing them. This highlights the importance of considering the behavior of both participants, as well as their biomechanics and ecology, in developing a more integrative understanding of organismal interactions.
\end{abstract}

Key words: predation, insect flight, dragonflies, Drosophila.

\section{Introduction}

Predation is one of the fundamental ways in which organisms interact. This interaction is interesting from both a biomechanical and an ecological perspective. Biomechanically, predator-prey encounters may be one of the most challenging behaviors that animals engage in, consisting of rapid sequences of complex, unsteady maneuvers that both participants are highly motivated to perform well. In addition, predation is a strong driving force for the evolution of morphological and physiological traits; thus, examining the mechanics of predation may reveal selective pressures underlying key structure-function relationships.

Ecologically, predation often plays an important role in shaping community structure through its effects on predator-prey population dynamics and species diversity (e.g. Paine, 1966; Hanski et al., 2001). It also has clear consequences for individual fitness, although the strength of selective forces acting on predator vs prey may differ; failure for the predator simply results in a missed meal whereas for the prey the consequences are much more dire [i.e. 'the life-dinner principle' coined by Dawkins and Krebs (Dawkins and Krebs, 1979)].

Despite the fact that the outcome of each encounter is not as critical for the predator as for the prey, overall performance and success rate may still be crucial for many predators. For example, adult odonates (dragonflies and damselflies) emerge with empty guts and minimal fat but both sexes gain mass rapidly during adulthood, often doubling their weight in a matter of weeks (Anholt et al., 1991). In females, mass gained through prey capture is a direct determinant of fecundity, and in males, mass gain increases flight muscle mass, which is correlated with reproductive success. Yet foraging also appears to carry an inherent risk of mortality, presumably because hunting odonates more often fall prey to predators themselves (Anholt, 1991).

Most studies of predation adopt one of two approaches, focusing on either the outcome of predation or the mechanics of the predator-prey interaction itself. The former approach is common in ecological studies, where the outcome of numerous encounters can be assessed either directly (e.g. by measuring capture rates in the field) or indirectly (e.g. by measuring survival rates of prey or analyzing gut contents of predators). These types of studies can provide insight into how abiotic factors and other features of the environment affect predation, as well as how capture or evasion rates vary between individuals, populations and species. However, the underlying morphological, physiological and behavioral traits that determine the outcome of each interaction can rarely be resolved. Even in systems where the location and timing of predation encounters is predictable, it can be difficult to perform repeated observations on the same individual, and to collect the high-resolution morphological and kinematic data necessary to form a mechanistic understanding of the factors contributing to capture or evasion.

The latter approach to studying predation, in which the mechanistic details of the predator-prey encounter itself are analyzed, is more common in laboratory-based studies examining the relative locomotory biomechanics and/or sensory capabilities of predator and prey. These types of studies provide more control over the timing and location of predatory encounters, and often 
elicit predatory or escape behaviors by using artificial or simplified stimuli to reduce variability. The controlled setting allows for repeated trials on known individuals and high-resolution data collection but is limited by the fact that many wild animals cannot be maintained in laboratory conditions or will not perform natural predatory behaviors in these environments.

Foraging modes of predators vary along a continuum of strategies from ambush to active predation. Ambush predators typically hide and wait for unsuspecting prey to approach, then attack in a rapid burst of movement, accelerating all or part of their body to capture prey, whereas active predators engage in prolonged searches and/or chases to locate and subdue prey. Ambush predation, which often consists of a rapid predatory strike that stimulates a prey escape response, occurs over relatively short spatial and temporal scales and can be elicited more easily than prolonged, active chases that cover large areas. This type of predation encounter is thus more amenable to laboratory settings, and has been the subject of numerous studies, particularly in aquatic systems.

Most of these studies focus on only one of the participants, quantifying the mechanics of predatory strikes (e.g. Nyberg, 1971; Webb and Skadsen, 1980; Rand and Lauder, 1981; Higham, 2007), fast-start escape responses (e.g. Webb, 1976; Domenici and Blake, 1997; Meager et al., 2006) or occasionally the mechanics of both behaviors in the same organism (Harper and Blake, 1991; Schreifer and Hale, 2004). In many cases, artificial stimuli such as electrical shock or model predators are used to elicit escape responses, and tethered prey are used to elicit predatory strikes. Even in studies where live, free-swimming predators and prey are used, typically the performance of only one participant is quantified (Webb, 1984; Webb, 1986; Juanes and Conover, 1994); studies that quantify the locomotory performance of both predator and prey are rare (Walker et al., 2005).

In aerial systems, far fewer studies have addressed the mechanics of predation, perhaps because short-term predatory strikes are less common than prolonged chases in aerial animals. Several studies have quantified the escape performance of flying insects but this behavior is usually motivated by artificial or atypical stimuli (e.g. Yager and May, 1990; Srygley and Kingsolver, 2000; Almbro and Kullberg, 2008; Jantzen and Eisner, 2008; Combes et al., 2010), or rarely by a trained predator in pursuit (Srygley and Dudley, 1993). The flight performance of a predator and prey each flying alone under laboratory conditions has been compared (McLachlan et al., 2003), and the flight paths of predators and their prey have been reconstructed to assess interception strategy (Olberg et al., 2000; Ghose et al., 2006; Ghose et al., 2009) but studies of the simultaneous flight mechanics of both participants during natural predatory encounters are virtually non-existent.

Motivated by the potential benefits of combining an ecological field-based approach with a detailed, mechanistic study of predator-prey interactions, we developed a system in which natural, complex interactions between dragonflies and their prey can be elicited repeatedly in captive individuals. This allows us to analyze predator-prey flight mechanics at high spatial and temporal resolution, as well as to quantify individual capture success by observing large numbers of interactions over extended periods of time. Our design builds on previous work by Olberg et al., which established a technique of eliciting predatory behavior in captive dragonflies (who normally will not forage in captivity) by housing them in a large outdoor, netted enclosure (Olberg et al., 2007). We used a similar method; we introduced wild dragonflies to a large greenhouse habitat and performed predation trials by releasing prey near perched dragonflies while capturing high-resolution films of predator-prey interactions and quantifying capture success over periods ranging from several days to several weeks.

One of our goals in combining these approaches was to gain insight into the physiological and behavioral adaptations that allow predators and prey to co-exist. Because the outcome of predator-prey encounters can have a strong effect on fitness, on an evolutionary timescale, predators and their prey are involved in an escalating arms race (Dawkins and Krebs, 1979); as one contestant improves performance, the other must adapt as well in order for the relationship to remain stable. Thus, we would expect that if one participant is faster, the other must excel at something else, either a locomotory trait such as acceleration or maneuverability, or a behavioral trait such as strategy, stealth or responsiveness. If this situation did not exist, the inferior contestant would eventually die out.

In many predator-prey contests, one of the participants (often the predator) is larger, and velocity typically scales with body size (Domenici, 2001; Alerstam et al., 2007; Vogel, 2008). In the case of dragonflies and their prey (e.g. small Dipterans such as mosquitoes or fruit flies), the size difference is substantial, with dragonflies dwarfing their prey by a length ratio of $\geq 20: 1$ and a mass ratio of $\geq 300: 1$. Combined with the greater relative force production capacity of dragonflies [due to a high flight muscle-tobody mass ratio (Marden, 1989)], this can be expected to lead to a substantial velocity difference. The size scaling of velocity favors larger predators, and raises the question of how smaller, slower prey are able to escape. Many studies in aquatic systems have proposed that while larger aquatic predators can reach higher velocities, their prey are capable of greater acceleration and/or maneuverability, and thus are able to escape some (or even most) of the time (Webb and de Buffrénil, 1990; Domenici, 2001; Maresh et al., 2004). Thus, in some groups of animals, locomotor traits appear to scale differentially with size, so that while certain aspects of locomotory performance (such as velocity) improve with body size, others decline - providing smaller, slower prey with a means of escape.

However, the evidence for scaling relationships among these other locomotory traits is not as strong as for velocity. While theoretical arguments and some data suggest that acceleration ability decreases with size in fish and aquatic vertebrates (Webb and de Buffrénil, 1990; Domenici, 2001), other studies show that acceleration is size-independent in a broad range of aquatic organisms (Webb, 1976; Vogel, 2008). In flying animals, maximum acceleration during take-off or vertical ascent decreases with size in some groups of birds (Warrick, 1998; Tobalske and Dial, 2000; Dial et al., 2008), but vertical acceleration during load lifting does not show the same relationship (Marden, 1994; Chai and Millard, 1997).

Quantifying maneuverability is less straightforward than measuring velocity or acceleration. The measurements most commonly employed are the turning rate (measured in degrees per second) and the radius of curvature of an animal's path (where a smaller radius of curvature indicates a tighter turn). In fish and cetaceans, it has been shown that turning rate generally declines with size and turning radius increases with size (Webb, 1976; Domenici, 2001; Blake and Chan, 2006), suggesting that smaller fish are more maneuverable overall. However, neither of the above measurements accounts for the forward velocity of the animal, and thus they may be more relevant for short-term behaviors starting from rest (such as predatory strikes and fast-start escape responses) than for prolonged, active chases where forward velocity may impose an additional constraint on an animal's turning ability. 
The influence of forward velocity in determining whether prey can evade predators via a sharp turn at the last minute (the "turning gambit') was described mathematically by Howland, who proposed that in order for the prey to escape the predator, its normalized velocity must be greater than the square root of its normalized turning radius (Howland, 1974). Later studies have used this relationship to predict whether various prey should be able to escape from predators via a turning gambit (Hedenström and Rosén, 2001). However, while this theoretical model provides a motivating framework for experimental studies of predator-prey encounters, its applicability to most predator-prey systems is probably limited due to the assumptions of steady forward velocity and motion in a single plane.

In addition to differential scaling of locomotor traits, a number of alternative hypotheses could explain how smaller, slower prey are able to co-exist with larger, faster predators, such as camouflage or stealth, erratic or unpredictable locomotory behavior, or the exploitation of refugia that exclude predators. The relative sensory capabilities of predator and prey, as well as the neural processing time from detection to motor response, are likely to play a key role in many predator-prey encounters as well.

Unfortunately, our ability to evaluate the relative importance of these factors in determining the outcome of predator-prey encounters is hampered by a lack of data. While numerous studies have examined the scaling of locomotory performance, measurements of maximal performance are rare, and our mechanistic understanding of predator-prey encounters remains much as Howland described it nearly four decades ago, where '... the essential data, namely simultaneous measurements of the speeds and turning radii of frequent predator-prey combinations are, to my knowledge, nowhere available' (Howland, 1974).

In this study, we combined an ecological approach, quantifying prey capture success and identifying factors that contribute to its variability, with a mechanistic approach, in which the biomechanics of individual predator-prey interactions were analyzed in detail. We measured daily capture success in nine dragonflies over 4-15 days per individual, and assessed the effects of abiotic environmental variables and prey density on success rate. We also recorded 50 three-dimensional, high-speed videos of predation encounters and analyzed the simultaneous flight biomechanics of predator and prey. The results revealed that locomotory performance, sensory capabilities and individual differences in behavior all play a role in determining the outcome of predator-prey interactions, and that complementary ecological and mechanistic approaches can help identify key factors governing complex interactions between animals.

\section{Materials and methods}

Insect handling and dragonfly predation trials

Dragonflies (Libellula cyanea Fabricius) were captured at a pond in Bedford, MA, USA, during the summer of 2010, numbered and released into a large greenhouse enclosure $(7.3 \times 7.3 \times 4.6 \mathrm{~m}$ high). The greenhouse contained shrubbery and artificial ponds, and was enclosed by wide mesh screening that allowed small prey, as well as natural light and air currents to enter the habitat (Fig. 1A). Dragonflies were allowed to acclimate to the greenhouse for at least one day, and most individuals rapidly began perching and feeding on prey (Fig. 1B). Naturally occurring prey were supplemented by fruit flies (Drosophila melanogaster Meigen) reared on standard fruit fly medium in large containers ( $\sim 2$ liters) and released into the greenhouse, where they were attracted to rotting fruit distributed throughout the habitat.
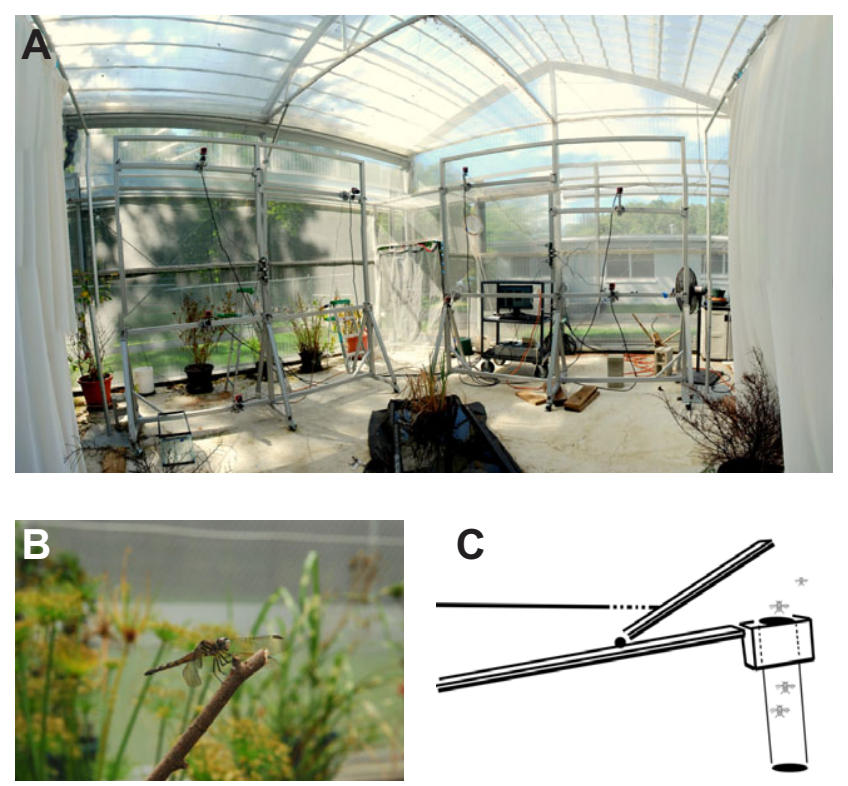

Fig. 1. Artificial dragonfly habitat and equipment used for conducting predation trials. (A) Photograph of the artificial habitat showing two orthogonal camera racks, foliage and pond. (B) Photograph of a dragonfly perched in the artificial habitat. Prey were released near perched dragonflies to initiate predation encounters. (C) Drawing of the mechanism used to release prey during trials. Fruit flies were place in a vial with a remote release mechanism, allowing the interaction to be initiated from $>1 \mathrm{~m}$ away. The top of the vial was opened remotely and several flies were allowed to emerge on their own, without tapping or shaking the vial.

From June to August 2010, we conducted $>2500$ predation trials on nine individual dragonflies, and recorded temperature, ultraviolet (UV) light intensity (power from 280-400 nm in $\mathrm{mW} \mathrm{cm}^{-2}$ ) and visible light intensity (unweighted total power over the visible spectrum in $\mathrm{W} \mathrm{m}^{-2}$ ) every hour throughout each day. During feeding trials, we released fruit flies near perched dragonflies by opening the top of a vial containing flies with a remote release mechanism (Fig. 1C) that permitted us to initiate the interaction from a distance $(\sim 1.1 \mathrm{~m})$. Fruit flies were allowed to emerge on their own, without tapping or shaking the vial. Because not all Drosophila will fly voluntarily, and emerging flies did not always head towards the perched dragonfly, we placed a number of flies in the vial at any one time. Upon opening the top, some fraction of the flies in the vial emerged; the perched dragonfly initiated a chase if one or more flies entered its feeding range. We performed trials at two levels of prey density: low-density trials in which $\sim 5-10$ flies were placed in the vial, and high-density trials in which $\sim 15-25$ flies were placed in the vial.

A chase was scored as successful if mastication was observed after the dragonfly returned to its perch. When at least 30 trials were recorded for an individual in one day, daily success rate was calculated as the number of successful chases divided by the total number of attempts. Temperature, UV and visible light intensity associated with each success rate were calculated by averaging hourly values recorded during the time over which trials were conducted.

Success rates were transformed with an arcsine square-root transformation to achieve normality for statistics (Kolmogorov-Smirnov test, $P=0.321$ ). The effects of individual dragonfly and prey density on transformed daily success rate were analyzed with an ANOVA in SPSS 18 (SPSS Inc., Chicago, IL, 
USA). The effects of temperature, UV and visible light on success rate across all trials were analyzed by stepwise regression, followed by stepwise regression analysis within individual dragonflies that had eight or more measurements of daily success rate.

\section{High-speed filming of predator-prey encounters}

We recorded $\sim 450$ predation trials with high-speed video, using multiple, synced video cameras (Photonfocus MV1-D1312-160CL-12, 8-camera system, Lachen, Switzerland) filming at 108 frames s$^{-1}$ to provide at least three views of each predation attempt. Shutter speeds varied from $\sim 4-6 \mathrm{~ms}$, depending on light levels, and motion blur was minimal due to the abundant natural light. The cameras were arranged on two sides of a filming area $\sim 3 \times 3 \times 2.5 \mathrm{~m}$ high in the center of the greenhouse (Fig. 1A), and white curtains were extended on the other two sides to reduce background clutter in the videos.

This relatively large filming area provides two benefits. First, because the direction and extent of predation trials is unpredictable, filming a large three-dimensional space with numerous cameras ensures that we will obtain at least three views of the predation attempt no matter where it occurs. Second, by initiating predation attempts in the center of this large area, we are able to avoid problems associated with analyzing data from the edges of our calibrated space (as most linear transformation methods are unable to fully account for the effects of lens distortion). Of the 450 filmed trials, we chose 50 to analyze in which the fruit fly was clearly visible from before or shortly after dragonfly take-off through to the conclusion of the interaction.

Three-dimensional flight paths of the dragonfly and prey were digitized from videos using DLTdv5 (Hedrick, 2008) in MATLAB R2009b (MathWorks, Natick, MA, USA), and the filming space was calibrated frequently by tracking the position of a wand of known length as it was moved throughout the airspace (using a calibration routine courtesy of T. Hedrick). The spatial accuracy of our system was estimated by applying the resulting calibration to the original videos of the moving wand and comparing estimated wand length with actual length. We also verified the accuracy of our system by dropping a small rubber ball in the filming area, tracking its position and calculating vertical acceleration during free fall (using the same techniques as for the insects, described below), then comparing these estimates with gravitational acceleration $\left(9.8 \mathrm{~m} \mathrm{~s}^{-2}\right)$. We checked for spatial bias in our kinematic analyses by performing an ANOVA on vertical acceleration estimates for the ball dropped in six different locations throughout the filming area.

\section{Analysis of predator-prey flight biomechanics}

Our multi-camera system filming at 108 frames s$^{-1}$ provided high temporal resolution of the three-dimensional trajectories of dragonflies and their prey (Fig. 2). This filming speed translated to an inter-frame interval of $\sim 9.3 \mathrm{~ms}$, and resulted in approximately five video frames per wing beat of the dragonflies (based on a flapping frequency of $\sim 22 \mathrm{~Hz}$ ) and approximately one frame per two wing beats of the fruit flies (based on a flapping frequency of $\sim 215 \mathrm{~Hz}$ ). Previous studies of dipteran flight mechanics suggest that several wing beats are required to elicit substantial changes in wing kinematic parameters (Balint and Dickinson, 2001), and that even rapid maneuvers such as saccadic turning require approximately $100 \mathrm{~ms}$ to complete [i.e. $>20$ wing beats for Drosophila (Tammero and Dickinson, 2002)]; thus, we believe that we are unlikely to miss any significant changes in Drosophila trajectory during the short interval between video frames.
We started digitizing body position of the dragonfly at take-off and of the fruit fly from the frame when it was first visible (generally at dragonfly take-off, sometimes slightly before or after) and continued to at least $100 \mathrm{~ms}$ after capture or the time step when dragonfly-fruit fly distance was minimal (in the case of failed attempts). Position data were filtered with a fifth-order low-pass Butterworth filter (utilizing zero-phase forward and reverse digital filtering) in Matlab. We performed a sensitivity analysis on several representative datasets of dragonfly and fruit fly kinematics to choose the most appropriate filtering frequency, which we determined to be $\sim 20 \%$ of the Nyquist frequency, or $22 \mathrm{~Hz}$; stronger filters (lower frequencies) typically resulted in large changes in peak velocity and maneuverability measurements whereas weaker filters (higher frequencies) resulted in dramatic changes in peak values, particularly for acceleration. We chose our cut-off value from within a small range of filter frequencies that produced relatively stable peak values of kinematic measurements for both dragonflies and fruit flies (e.g. peak values do not change dramatically as filtering frequency changes in either direction). This filtering frequency also falls close to the flapping frequency of dragonflies and thus eliminates within-wing beat motions of the dragonfly's body.

After filtering position data, we calculated velocity as the distance between successive three-dimensional positions divided by the time period, and acceleration as the difference between successive velocity measurements divided by time period. We defined each capture sequence as starting when the dragonfly took off and reached a velocity of $0.5 \mathrm{~m} \mathrm{~s}^{-1}$ and ending $\sim 100 \mathrm{~ms}$ (11 frames) after capture (or the time when the dragonfly and prey were closest in failed attempts). Within this period, we identified peak values attained by both dragonflies and their prey of velocity and acceleration, as well as deceleration, because deceleration in fluid media is governed more by inertia than muscle mass and thus may scale differently with size.

To quantify maneuvering, we calculated the two most commonly used measurements of maneuverability: turning rate (a measure of how quickly an animal turns), and radius of curvature (a measure of how sharply an animal turns, where a smaller turning radius indicates a tighter turn). Turning rate was calculated as the angular change in the direction of subsequent velocity vectors in threedimensional space $(\theta)$ divided by the time period. Radius of curvature $\left(r_{\text {curv }}\right)$ was calculated as the linear distance between an animal's position at every other frame (e.g. the shortest distance between positions at time steps 1 and 3 ) divided by $2 \times \sin (\theta)$ :

$$
r_{\text {curv }, \mathrm{i}}=\left|p_{\mathrm{i}+2}-p_{\mathrm{i}}\right| /\left[2 \sin \left(\theta_{\mathrm{i}}\right)\right],
$$

where $p$ is position, and $\theta_{\mathrm{i}}$ is the change in angle between velocity vectors $\mathbf{v}_{\mathrm{i}}$ and $\mathbf{v}_{\mathrm{i}+1}$.

As neither of these measurements accounts for forward velocity (and thus cannot reveal potential velocity-dependent constraints on turning performance), we also calculated the magnitude of radial acceleration $\left(a_{\mathrm{rad}}\right)$ of predator and prey as the product of subsequent velocity scalars divided by the radius of curvature:

$$
a_{\text {rad,i }}=\left(v_{\mathrm{i}} v_{\mathrm{i}+1}\right) / r_{\text {curv }, \mathrm{i}} \text {. }
$$

Finally, we identified peak turning rate and radial acceleration of each participant during the capture sequence, as well as their minimum radius of curvature.

To examine the relative performance of dragonflies and fruit flies during each encounter, we performed paired $t$-tests on the peaks of all six kinematic measurements (velocity, acceleration, deceleration, radial acceleration, turning rate and radius of 

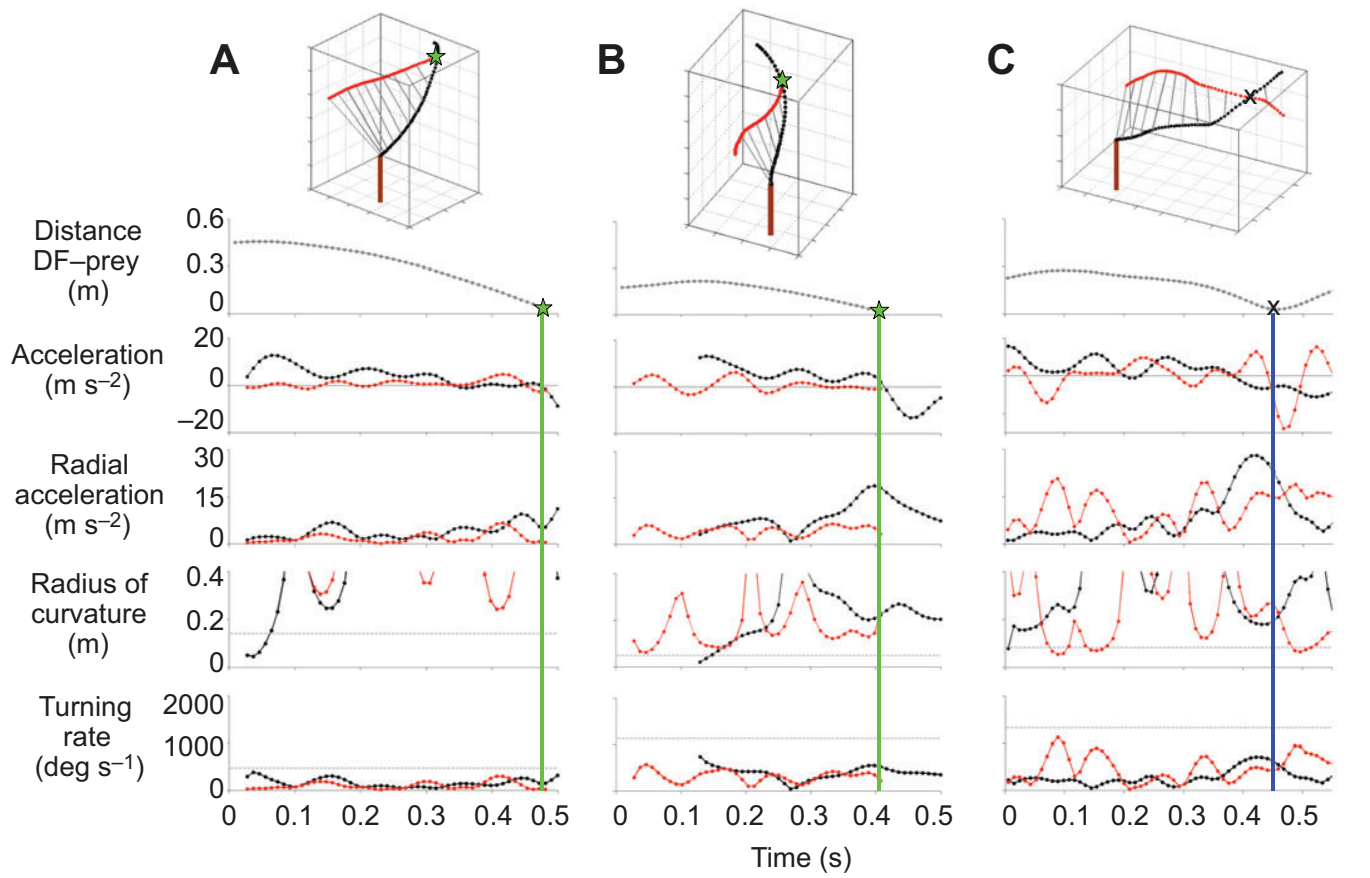

- Dragonfly

$\rightarrow$ Fruit fly

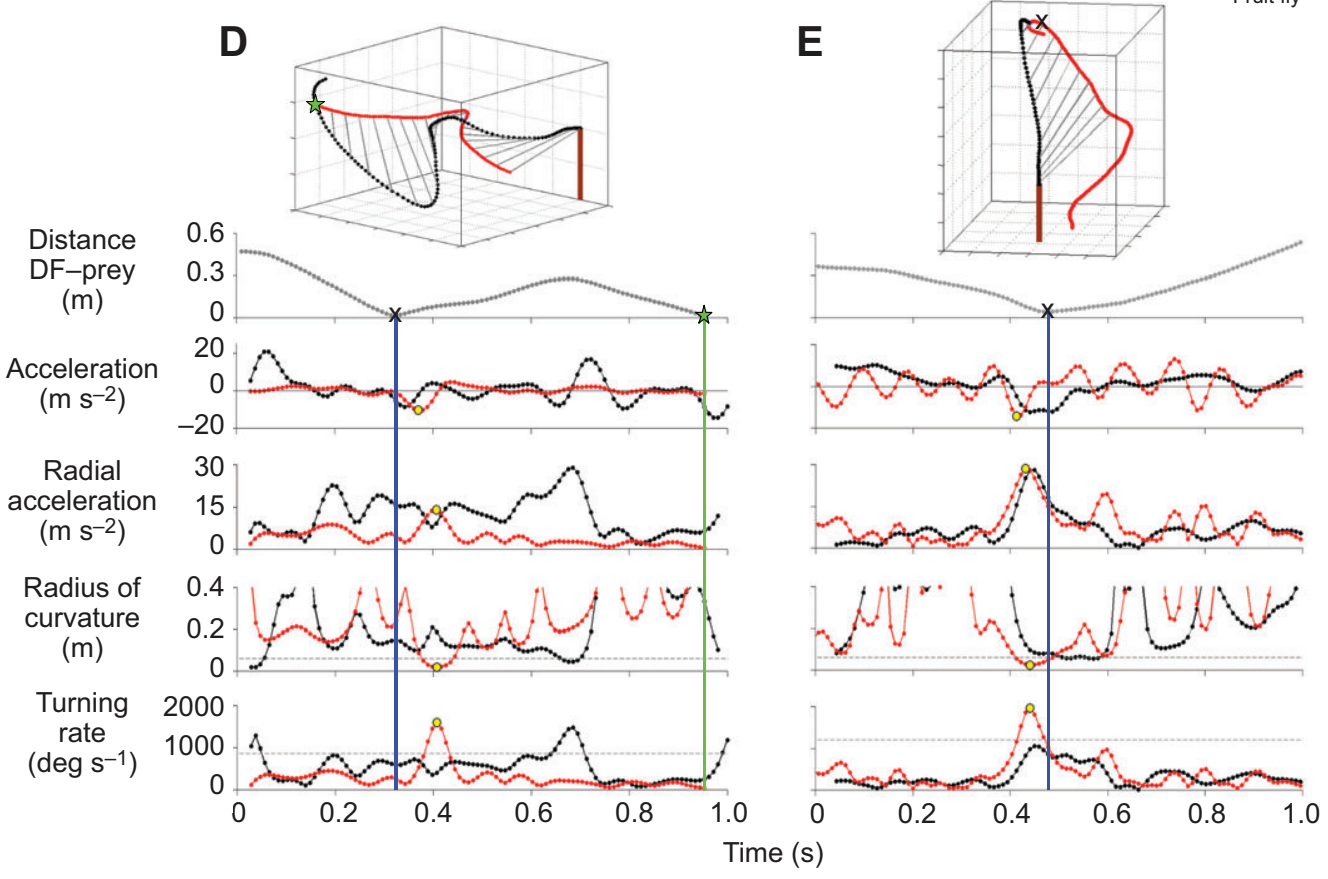

Fig. 2. Sample trajectories and flight kinematics of dragonflies and fruit flies during predation encounters. In each case, the three-dimensional trajectories of the dragonfly (black) and fruit fly (red) are shown, with gray lines connecting their positions every five frames, and the outcome of the capture attempt indicated by a green star (successful) or blue cross (failed). The location of the perch is shown in brown, and axis ticks represent a distance of $0.1 \mathrm{~m}$. Below the trajectories, five variables are plotted against time: the absolute distance between dragonfly and fruit fly (gray), tangential and radial acceleration, radius of curvature, and turning rate. Each time step is plotted (dragonflies in black and fruit flies in red), and vertical lines indicate a predation attempt (successful in green and failed in blue). The cut-off for classifying active evasive maneuvers is indicated on plots of turning rate (2.5 times median) and radius of curvature (median divided by 2.5) by a dotted gray line; peaks associated with evasive maneuvers are indicated by a yellow dot.

$(A, B)$ Predator-prey encounters in which fruit flies did not perform evasive maneuvers and captures were successful. (C) A

predator-prey encounter in which the fruit fly did not perform an evasive maneuver but the capture attempt failed, most likely due to erratic turning behavior of the prey. (D) A predator-prey encounter in which the dragonfly initially missed the fruit fly and the fly responded afterwards with an evasive maneuver; the dragonfly subsequently re-approached and successfully captured the fruit fly. (E) A predator-prey encounter in which the fruit fly performed an evasive maneuver as the dragonfly approached and successfully avoided capture. curvature). While a predation attempt may not elicit maximal performance from the dragonfly (as missing a meal is not a matter of life or death), we may be able to identify instances of maximal performance from the fruit flies, assuming that they are aware of the threat to their survival. Even though we are unlikely to elicit maximal performance from dragonflies, a comparison of peak kinematic variables over the 50 trials should still provide insight into the question of whether or not locomotory traits such as acceleration and maneuverability scale differentially with size; any potential scaling effects that provide a significant advantage to smaller animals should be readily apparent, even during sub-maximal flight performance, due to the large (i.e. several orders of magnitude) size difference between dragonflies and fruit flies.

\section{Distinguishing active evasion from routine maneuvers in fruit flies}

Because fruit flies typically explore their environment through a series of straight flight segments interspersed with rapid turns (or saccades) in random directions (Tammero and Dickinson, 2002), it is not immediately obvious which maneuvers (if any) represent active evasive responses to an approaching dragonfly, as opposed to routine flight behavior. To aid in identifying evasive responses during predation trials, we performed an additional set of experiments in which we released fruit flies in the greenhouse using all of the same methods as during predation trials but with no dragonfly present. We also filmed a series of fruit fly releases inside the laboratory in an area of $\sim 2 \times 2 \times 2 \mathrm{~m}$ (calibrated and verified using the same methods as for the outdoor filming area) to compare 
Table 1. Variability in daily success rates of hunting dragonflies

\begin{tabular}{ccccccc}
\hline ID & N days & Median success rate & Range success rate & Model variable & $R^{2}$ & $P$ \\
\hline 1 & 15 & 0.900 & 0.161 & Visible light & 0.363 & $0.017^{\star}$ \\
2 & 15 & 0.878 & 0.334 & Visible light & 0.579 & $0.001^{* *}$ \\
3 & 14 & 0.952 & UV light & 0.052 & 0.454 \\
4 & 9 & 0.833 & Visible light & 0.316 & 0.115 \\
5 & 8 & 0.934 & 0.233 & Visible light & 0.024 & 0.712 \\
6 & 8 & 0.917 & 0.233 & Visible light & 0.836 & - \\
7 & 5 & 0.933 & 0.219 & - & - & - \\
8 & 5 & 0.839 & 0.169 & - & - & - \\
9 & 4 & 0.917 & 0.174 & - & 0.068 & $0.018^{*}$ \\
All & 83 & 0.921 & 0.367 & Visible light & \\
\hline
\end{tabular}

For each individual, the number of days over which success rates were measured, median and total range of daily success rates, and results of stepwise regression analysis with temperature, ultraviolet (UV) and visible light level are reported. Regression analysis was not performed when less than eight daily measurements were available. The final row reports data for all individuals combined. Asterisks indicate statistical significance at the $P<0.05\left({ }^{*}\right)$ or $P<0.01$ $\left({ }^{* *}\right)$ level.

the flight behavior of Drosophila in our outdoor greenhouse vs the indoor laboratory conditions under which Drosophila flight is typically studied. These indoor and outdoor prey-only releases were all performed on the same day using laboratory-reared flies taken from the same container.

We also performed a more detailed analysis of Drosophila flight kinematics during predation trials to determine typical characteristics of cruising flight and individual variability in flight behavior between flies. We identified individual turns by finding all peaks in turning rate and radial acceleration, along with all minima in radius of curvature, during the entire sequence (a local maximum was considered a peak if its value was at least 1.3 times the value of the minima preceding and following it). We then calculated the median and interquartile range of turning rate, radial acceleration and radius of curvature for each flight sequence that contained four or more peaks. Evasive responses were identified by searching for correlated peaks in turning rate, radius of curvature and radial acceleration that were significantly larger than the median value of these variables within that flight sequence (further specifics provided below).

\section{Results}

\section{Daily predation success rates in dragonflies}

We were able to measure predation success rate over multiple days in nine dragonflies feeding on fruit flies. Overall capture success was high and similar between individuals, with median success rates ranging from 0.83 to 0.95 ; however, daily capture success varied widely within individuals, with a mean range of 0.22 and a maximum of 0.37 (Table 1 ). The density of prey had a significant effect on capture success, with a higher density of prey leading to greater success (ANOVA, prey density: $F=7.261, P=0.001$ ). There was no statistically significant difference in success rate between individuals and no interaction between individual and prey density (ANOVA, individual: $F=1.537, P=0.164$; individual $\times$ prey density: $F=0.855, P=0.595)$.

Stepwise regression analysis removed temperature and UV light as non-significant factors in determining daily success rate, and produced a significant model with visible light intensity as the only explanatory factor $(P=0.018)$, although light intensity explained less than $7 \%$ of the variability in success rate across trials $\left(R^{2}=0.068\right)$. One reason for this may be that the effect of light intensity on predation success appears to vary between individuals. Within-individual stepwise regression analysis revealed that visible light had a significant effect on success rate in only half of the individuals examined (with $R^{2}$ varying from 0.363 to 0.836 ) whereas light had no apparent effect on predation success in the remaining individuals (Table 1 ).

\section{Flight biomechanics of dragonflies and fruit flies}

The test analysis of a falling ball showed that we were able to estimate downward acceleration to within $5 \%$ of its true value on average (maximum error $=7.9 \%$ ), and that these acceleration estimates were not significantly affected by location (ANOVA, drop location: $F=0.792, P=0.536$ ). Comparisons of the estimated vs actual length of our calibration wand that was moved throughout the filming space in nine calibration videos produced a mean spatial accuracy estimate of $3.9 \mathrm{~mm}$ (minimum $=1.6 \mathrm{~mm}$, maximum $=7.2 \mathrm{~mm}$ ) or $\sim 1.3-1.5$ Drosophila body lengths (depending on sex). This is a very conservative estimate of our spatial accuracy for two reasons. First, these values are based on the total error in our estimate of wand length and represent the worst case scenario [where we assume all of the error in wand length was due to incorrectly localizing one end of the wand (with the other end localized perfectly)], as opposed to the more likely scenario that localization error was equal at both ends (in which case our spatial accuracy estimates should be reduced by half and would be approximately $2 \mathrm{~mm}$ or $<1$ Drosophila body length). Second, this estimate is based on mean error in wand length as the wand is moved throughout our filming area. Linear transformations nearly always perform worst near the edges of the calibrated space due to lens distortion. However, the 50 predator-prey flight trajectories analyzed here never approached the edges of the calibrated airspace; most trajectories remained within the inner third of the filming area (with buffer zones of $0.75-1.1 \mathrm{~m}$ on each side, $1 \mathrm{~m}$ on bottom and $0.5-1 \mathrm{~m}$ on top), and thus occurred within a region where we were likely able to track position more accurately than what was reflected by the spatially averaged error estimate.

Dragonflies successfully captured their prey in 43 of the 50 trials analyzed. The majority of captures occurred in less than half a second (mean $=0.44 \pm 0.18 \mathrm{~s}$ ) and required the dragonfly to travel a little over half a meter from its perch (mean $=0.60 \pm 0.26 \mathrm{~m}$ ). All captures occurred at a vertical position above the dragonfly's perch. In general, flight kinematics of both the predator and prey were highly variable between trials (Fig. 2); however, dragonflies typically reached peak acceleration shortly after take-off (mean time, normalized as the proportion of time from take-off to capture $=0.27 \pm 0.26$ ) and peak velocity shortly before capture (mean normalized time $=0.84 \pm 0.21$ ). 

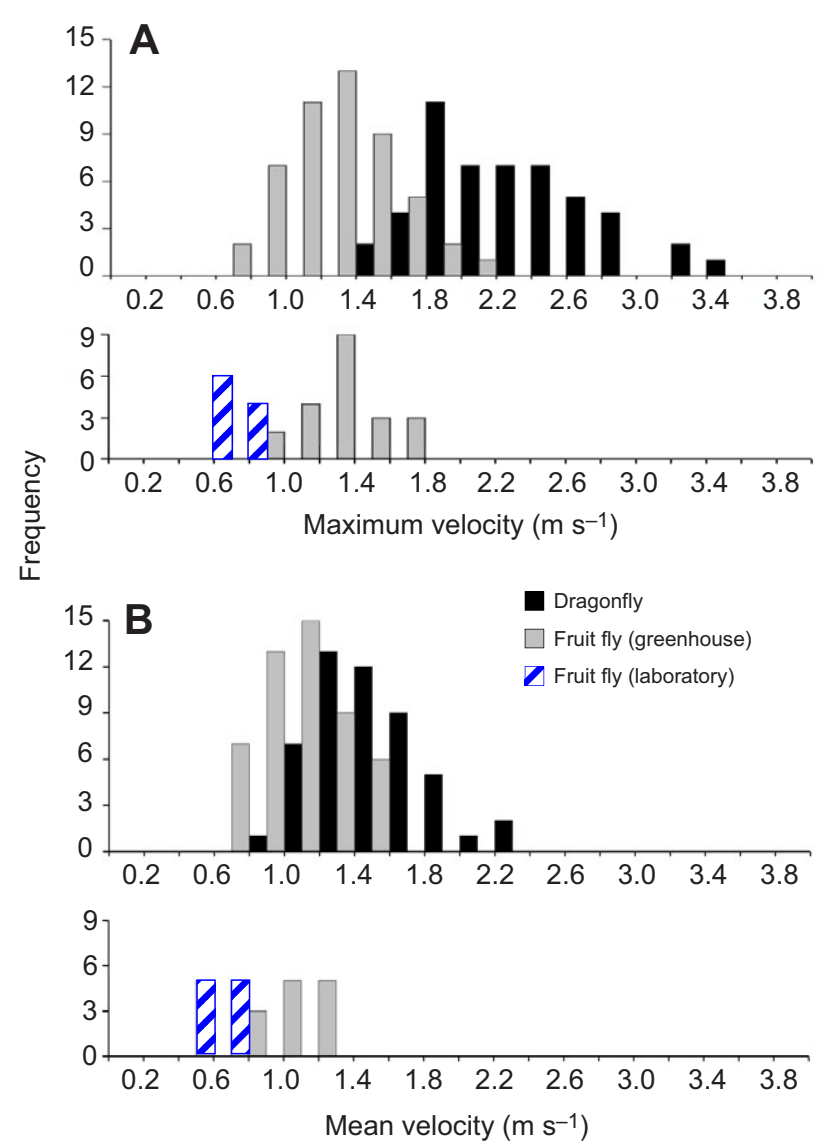

Fig. 3. Distribution of flight velocities in dragonflies and fruit flies. In each case, the top panel shows the velocities of dragonflies (black) and fruit flies (gray) during the 50 predator-prey encounters. The bottom panel shows the velocities of fruit flies during prey-only releases in the greenhouse (gray) or in the laboratory (blue stripes). (A) Distribution of maximum flight velocities. (B) Distribution of mean flight velocities.

Maximum velocity of dragonflies was always higher than the maximum velocity of their prey (paired $t$-test, $P<0.001$; Table 2 ), and peak dragonfly velocity was strongly related to peak prey velocity $\left[v_{\text {max,dragonfly }}=\left(1.5527 \times v_{\max , \text { prey }}\right)+0.604, R^{2}=0.5851\right]$. The peak velocities measured for Drosophila in this experiment $\left(0.78-2.03 \mathrm{~m} \mathrm{~s}^{-1}\right.$; Fig. 3A) were somewhat surprising given previously reported values for maximum flight speed in Drosophila, which typically range from $0.7 \mathrm{~m} \mathrm{~s}^{-1}$ to $1.2 \mathrm{~m} \mathrm{~s}^{-1}$ (e.g. Marden et al., 1997; Montooth et al., 2003; Mronz and Lehmann, 2008; Fry et al., 2009). However, all of these previous studies were performed in laboratory settings where temperatures and light levels are lower, flight arenas are smaller, and visual and olfactory cues differ from those in our greenhouse.

To determine whether this discrepancy between our peak Drosophila flight velocities and previously reported values is due to the experimental setting, we compared peak velocities from the Drosophila-only releases performed in the greenhouse and in the laboratory on the same day. Peak velocity of the outdoor releases (mean $=1.31 \pm 0.25 \mathrm{~m} \mathrm{~s}^{-1}, N=21$ ) did not differ from peak velocity during predation trials $(t$-test, $P=0.938)$ but it was significantly higher than peak velocity of the indoor releases (mean $=0.79 \pm 0.08$, $N=10$; $t$-test, $P<0.001$; Fig. $3 \mathrm{~A}$ ). Mean flight velocities showed the same relationship (mean outdoor $=1.07 \pm 0.19 \mathrm{~m} \mathrm{~s}^{-1}$, mean indoor $=0.60 \pm 0.10 \mathrm{~m} \mathrm{~s}^{-1}$; $t$-test, $P<0.001$; Fig. $3 \mathrm{~B}$ ).

In addition to reaching higher peak velocities during predation encounters, dragonflies significantly outperformed fruit flies in maximum acceleration, deceleration and radial acceleration, as well as minimum radius of curvature (Table 2; Fig. 4A-D). Peak turning rates were not significantly different between dragonflies and fruit flies (Table2), although in several cases fruit flies reached turning rates higher than the maximum rates observed in dragonflies (Fig. 4E).

Our detailed analysis of Drosophila flight behavior revealed a strong relationship between radius of curvature and turning rate that is maintained over turns spanning several orders of magnitude in strength (Fig. 5A). Despite this consistency in turning characteristics, individual fruit flies varied enormously in the typical strength of turns performed during flight (with median turning rates ranging from $115.0 \mathrm{deg} \mathrm{s}^{-1}$ to $665.7 \mathrm{deg} \mathrm{s}^{-1}$ and median radii of curvature ranging from $0.097 \mathrm{~m}$ to $0.475 \mathrm{~m}$; Fig. $2 \mathrm{~A}-\mathrm{C}$, Fig. 5B) and in the variability of their turning behavior (with turning rate interquartile ranges ranging from $26.2 \mathrm{deg} \mathrm{s}^{-1}$ to $552.0 \mathrm{deg} \mathrm{s}^{-1}$; Fig. 5B).

To determine which (if any) of these turns were evasive responses elicited by an approaching dragonfly, we first examined two trials in which a fruit fly displayed obvious peaks in turning rate and radius of curvature after a dragonfly passed nearby but failed to capture it (e.g. Fig. 2D). We calculated the ratios of these peaks to the median values of turning rate and radius of curvature for each trial and found that these ratios were high in both cases (3.4 and 4.6 for peak:median turning rate; 8.4 and 8.2 for median:minimum radius of curvature). In addition, both maneuvers were associated with the maximum peak in radial acceleration, as well as maximum peaks in acceleration and/or deceleration for each trial (e.g. Fig. 2D).

We next calculated peak:median ratios of turning rate and radius of curvature, and examined correlated peaks in radial and tangential acceleration during the most extreme turn in each of the 48 remaining trials. We found that the peak:median ratios for most trials were within 2.5 times the median value of turning rate and

Table 2. Relative flight performance of dragonflies and their prey

\begin{tabular}{|c|c|c|c|c|}
\hline Kinematic variable & Dragonfly (means \pm s.d.) & Fruit fly (means \pm s.d.) & Paired $t$-test $(P$-value $)$ & Relative performance \\
\hline Velocity $\left(\mathrm{m} \mathrm{s}^{-1}\right)$ & $2.28 \pm 0.46$ & $1.30 \pm 0.30$ & $<0.001^{\star \star \star}$ & $\mathrm{DF}>\mathrm{FF}$ \\
\hline Acceleration $\left(\mathrm{m} \mathrm{s}^{-2}\right)$ & $14.9 \pm 3.9$ & $5.5 \pm 4.7$ & $<0.001^{\star \star \star}$ & $D F>F F$ \\
\hline Deceleration $\left(\mathrm{m} \mathrm{s}^{-2}\right)$ & $-14.8 \pm 4.2$ & $-6.8 \pm 6.2$ & $<0.001^{\star \star \star}$ & $\mathrm{DF}>\mathrm{FF}$ \\
\hline Radius of curvature (m) & $0.041 \pm 0.024$ & $0.120 \pm 0.068$ & $<0.001^{* \star *}$ & $D F>F F$ \\
\hline Turning rate $\left(\operatorname{deg~s}^{-1}\right)$ & $914.0 \pm 325.5$ & $776.8 \pm 560.8$ & 0.08 & No difference \\
\hline
\end{tabular}

Means \pm standard deviations of peak values for each kinematic variable are shown ( $N=50$ trials). Results of paired $t$-tests assessing the difference between peak dragonfly and fruit fly performance in each encounter are shown, along with the relative performance of the participants (DF indicates dragonfly and FF indicates fruit fly). ${ }^{* \star *} P<0.001$. 

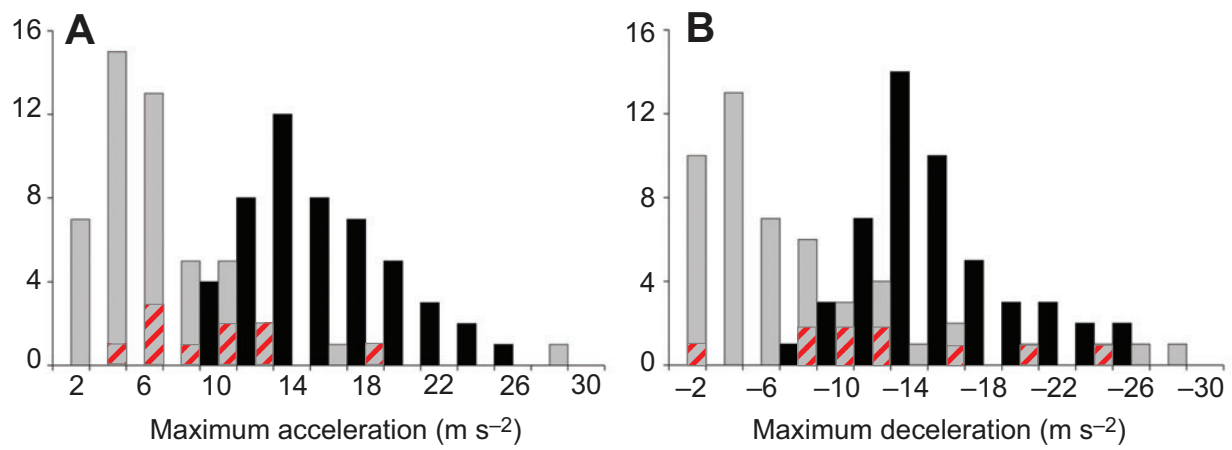

Fig. 4. Distributions of peak kinematic values in dragonflies and fruit flies during predation encounters. Dragonflies are shown in black and fruit flies in gray. Peak fruit fly values associated with active, evasive maneuvers are overlaid with red stripes. (A) Peak acceleration. (B) Peak (minimum) deceleration. (C) Peak radial acceleration. (D) Minimum radius of curvature, where smaller values indicate tighter turns. (E) Peak turning rate.
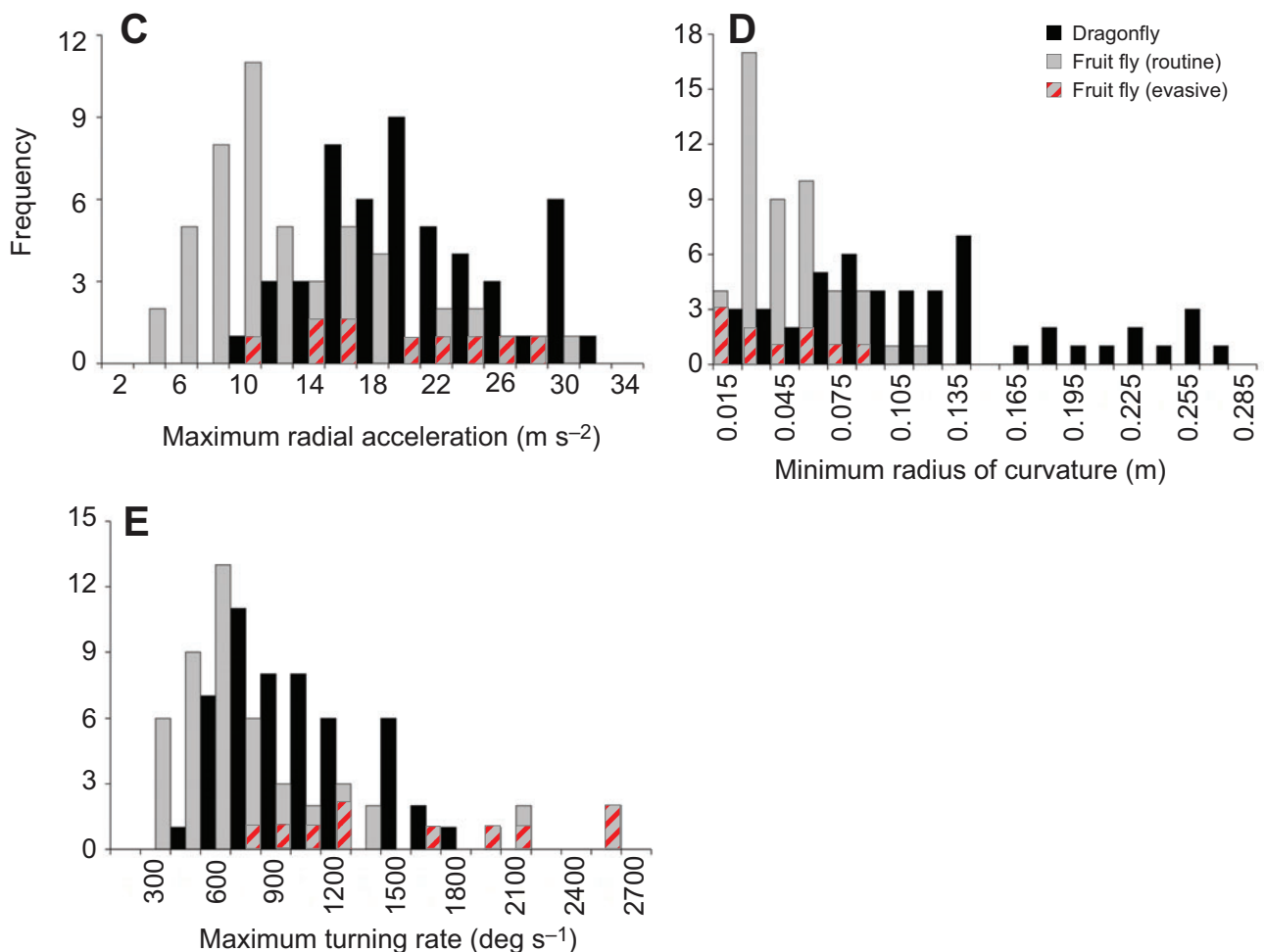

radius of curvature. However, we identified eight additional trials in which peak:median ratios of both turning rate and radius of curvature were more than 2.5 times greater than median values (usually significantly greater, e.g. Fig. 2E). All of these turns were also associated with a peak in radial acceleration (typically $>2.5$ times median value) and often with a minimum value of deceleration followed by a maximum acceleration. Based on these characteristics as well as the details of when these maneuvers occurred (i.e. the distance and position of the dragonfly relative to the fruit fly's presumed visual field), we are confident that these maneuvers should be classified as active evasive responses to the dragonfly. While this method may neglect to identify more subtle evasive responses that fruit flies might perform, we prefer to adopt a conservative approach to classifying maneuvers as active responses.

The evasive maneuvers identified using this method tend to lie near the higher end of the distribution of fruit fly performance measurements; however, because active responses are based on the height of peaks relative to each fly's typical turning behavior rather than the absolute value of peaks, not all of the highest performance values were classified as active responses (Fig. 4). Turning rate and radius of curvature were tightly correlated during evasive maneuvers, as during routine turns, but the slope of this relationship differed from that observed for typical turns (Fig. 5A).

\section{Discussion}

Insights gained from an ecological approach to studying predation By quantifying daily success rate in multiple individuals over thousands of predation trials, we were able to form a strong statistical understanding of the extent and sources of variability in dragonfly prey capture success. We found that dragonflies are highly effective predators of fruit flies, with median success rates ranging from 0.83 to 0.95 . While this high success rate may be partly due to the type of prey used in our studies, Libellulid dragonflies have previously been shown to be highly effective predators (with mean success rates ranging from 0.76 to 0.97 ), even when feeding in the field on a variety of wild prey (Baird and May, 1997; Olberg et al., 2000).

Despite the relatively consistent median success rates measured in our study, daily capture success within individuals varied widely. A portion of this variability can be accounted for by natural differences in visible light intensity, particularly in certain individuals who appear to be more sensitive to these changes. Solar radiation intensity was one of the few variables 


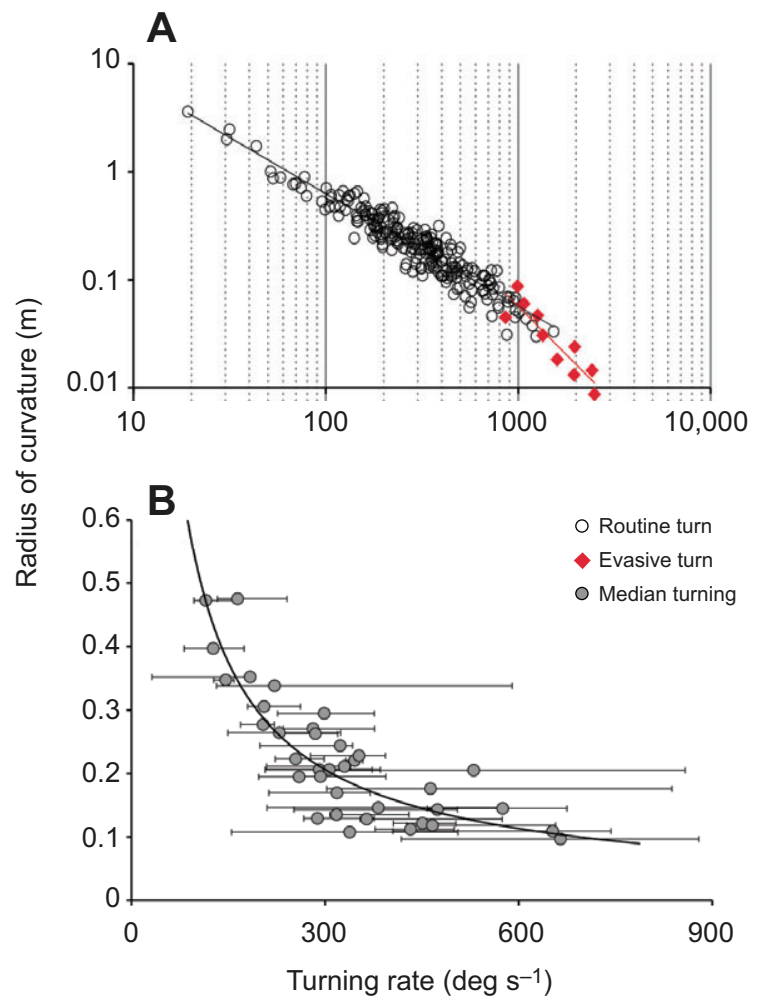

Fig. 5. Relationship between radius of curvature and turning rate during routine flight and evasive maneuvers in fruit flies. (A) Radius of curvature vs turning rate for all turns identified during predation trials. Turns performed during routine flight are indicated by open black circles $(y=75.679 x-1.0412$ $R^{2}=0.8908$ ), and turns associated with active maneuvers by closed red diamonds $\left(y=12784 x-1.7846, R^{2}=0.8176\right)$. (B) Median radius of curvature vs median turning rate for routine flight of fruit flies during predation trials. Interquartile ranges of turning rates are indicated by horizontal error bars. Values were only calculated for trials in which at least four distinct turns could be identified.

shown to have a significant effect on predation success in the field as well (Baird and May, 1997). This could contribute to the preferences frequently observed in dragonfly habitat choice (open $v s$ forested areas) and environmental conditions (sunny vs overcast days).

However, the strongest overall source of variability in capture success in our study was the density of prey. While this finding could have implications for how variations in natural prey density might affect the fitness of wild dragonflies, it is somewhat unsatisfying because it does not provide us with a mechanistic understanding of why increased prey density improves the capture success of dragonflies.

\section{Insights gained from a biomechanical approach to studying predation}

Simultaneous measurements of flight performance in dragonflies and their prey allowed us to definitively reject the hypothesis that negative scaling of acceleration or maneuvering flight performance allows smaller fruit flies to escape from larger, faster dragonflies, as dragonflies outperformed fruit flies in all locomotory traits except for turning rate (where there was no significant difference). If a significant scaling relationship existed, we would expect to see a large, obvious difference in performance, with fruit flies significantly outperforming dragonflies in at least one trait due to the large difference in body sizes.

Although there does not appear to be a significant underlying scaling effect in performance capabilities, it is still possible that the failed predation attempts we observed were in fact due to the fruit fly outperforming the dragonfly in those particular instances. In the seven failed predation trials we analyzed, peak locomotory performance of the fruit fly does sometimes exceed that of the dragonfly; however, there are only three cases in which peak performance of the fruit fly exceeds the maximum performance ever observed in the pursuing dragonfly over all of its filmed trials (and thus may indicate superior capability). In two of these cases, peak flight performance of the fruit fly actually occurred after the dragonfly passed nearby but failed to capture its prey; thus, in only one trial did we observe a peak in fruit fly performance (specifically in turning rate) that exceeded the apparent performance capabilities of the pursuing dragonfly, and which could potentially have contributed to the dragonfly's failure (as it immediately preceded the attempt).

All of the above hypotheses based on the relative locomotory performance of predator $v s$ prey contain an inherent assumption that the prey knows it is being pursued and responds accordingly. However, we find that the fruit fly seems to be unaware of the approaching dragonfly over $80 \%$ of the time (as obvious evasive responses preceding the capture attempt were absent in 42 of 50 trials). Dragonflies typically approach their prey from below, a strategy that allows them to track prey with high accuracy by maintaining the prey's image within a small, dorsal area of high visual acuity (Olberg et al., 2007). This strategy may provide the additional benefit of a stealthy approach, allowing dragonflies to capture unsuspecting prey and minimize the time, energy and exposure to predators associated with an extended chase of evasive prey.

Of the eight cases where fruit flies did appear to detect the approaching dragonfly before the capture attempt, half (four) of the resulting evasive responses were successful, but two of these successful evasions were immediately followed by the dragonfly re-approaching and successfully capturing the fly. Thus, fruit flies successfully evaded their pursuers and lived to tell the tale only $4 \%$ of the time.

This brings up the question of what caused the other five failed predation attempts. In one case, the cause of failure could not be determined, but the other four attempts appeared to have been thwarted by the routine, erratic turning behavior of the flies (flies performed a sudden routine turn or an unpredictable change in turning behavior as dragonflies were on their final approach). This highlights the importance of the typical flight behavior displayed by fruit flies and other Dipterans. The random turns performed during cruising flight not only allow flies to explore their environment thoroughly (locating mates or food sources and potentially detecting predators), but just by virtue of being erratic, this turning behavior may also allow flies to escape from approaching predators without even knowing it.

The unexpected results regarding the differences in indoor vs outdoor Drosophila flight velocities emphasize how important experimental conditions are in influencing behavior and in shaping our view of performance norms. While the ultimate cause of these differences is unknown, a number of factors could potentially explain the difference in flight speeds. Wind velocity is the least likely explanation, as air currents in the greenhouse were minimal during these experiments (any wind is rapidly attenuated by the mesh walls), and the flies' headings varied from trial to trial. Olfactory and visual cues (including the larger open space in the 
greenhouse) could potentially influence flight behavior. However, the most likely causes of these substantial differences in velocity are temperature, which affects muscle performance and other physiological functions, or light intensity, as dragonflies rely on vision to assess and track prey (Olberg et al., 2005; Olberg et al., 2007).

On the day we conducted the indoor and outdoor Drosophila releases, temperature differences between the greenhouse and laboratory were relatively small $\left(25.7^{\circ} \mathrm{C}\right.$ outside $v s 24.2^{\circ} \mathrm{C}$ inside $)$ but differences in light levels were substantial: UV light intensity was $31.5 \mathrm{~mW} \mathrm{~cm}^{-2}$ outside vs $0.037 \mathrm{~mW} \mathrm{~cm}^{-2}$ inside, and visible light intensity was $65,250 \mathrm{~cd} \mathrm{~m}^{-2}$ outside vs $857 \mathrm{cdm}^{-2}$ inside with standard overhead fluorescent lighting [note that this indoor lighting is even brighter than typical Drosophila flight test conditions, e.g. $183 \mathrm{cdm}^{-2}$ (reported in Mronz and Lehmann, 2008)]. Thus, the outdoor trials were more than 75 times brighter in the visible range and more than 850 times brighter in the UV range than the indoor trials, making light intensity a good candidate for explaining the large differences in flight behavior.

Finally, our analysis of Drosophila turning behavior during predation trials highlights the importance of performing experiments in an environment that elicits natural behaviors from both predator and prey, and of rigorously quantifying not only mean behavior but also variability within and between individuals. Although the turns performed during routine cruising flight appear to share certain characteristics (e.g. they conform to the same relationship between turning rate and radius of curvature; Fig. 5A), individual flies vary widely in both how strong and how variable these turns are (Fig.5B). Routine Drosophila turning behavior appears to have led to four failed predation attempts, and may have contributed to the flies' ability to detect the approaching dragonfly in cases where active evasive responses were observed (although only two of these resulted in a successful evasion). However, because of the low proportion of failed predation attempts and intensity of three-dimensional data analysis, our sample size of biomechanical analyses of failed predation attempts is too small to draw any definitive conclusions about how prey behavior relates to predation success from this data.

\section{Insights gained from combining ecological and biomechanical approaches}

Because our ecological approach to studying predation (quantifying variability in capture success over thousands of trials) provided strong evidence that dragonflies are significantly more successful hunters when prey density is high, we decided to re-examine our biomechanical data to see whether we could detect any differences in prey behavior resulting from low vs high-density releases. We were able to quantify routine flight behavior (i.e. identify at least four distinct turns) for 25 trials from low-density releases and 11 trials from high-density releases.

We found a significant difference in the variability of routine turning behavior between these groups ( $t$-test, $P=0.007)$, with flies from low-density releases displaying more variable turns (mean interquartile range of turning rate $=217.6 \mathrm{deg} \mathrm{s}^{-1} v s 112.2 \mathrm{deg} \mathrm{s}^{-1}$ in high-density releases), as well as a trend towards stronger turns in low-density releases (maximum turning rate of routine turns $=627.0 \mathrm{deg} \mathrm{s}^{-1}$ on average $v s \quad 475.1 \mathrm{deg} \mathrm{s}^{-1}$ in high-density releases; $t$-test, $P=0.06$ ). Thus, when prey density was high, the flies engaged in predatory encounters displayed more consistent flight patterns (less variable turning behavior) and tended to turn more slowly, and dragonflies were more successful at capturing them whereas when prey density was low, flies in predatory encounters displayed more erratic flight (more variable turning behavior) and tended to turn faster, and dragonflies were less successful at capturing them.

Combining the results of these two different approaches provides strong evidence that the routine flight behavior of individual prey is one of the most significant factors in determining the outcome of predatory encounters between dragonflies and fruit flies. The results do not reveal why the routine flight behavior of pursued flies from high $v s$ low-density releases differs, however. While it is possible that the number of flies interacting in the vial for several minutes before the release affects the flight behavior of emerging flies, it seems more likely that when prey density is high, dragonflies encounter a larger sampling of natural prey variability and are able to choose prey that are flying more predictably and thus are more attractive targets.

\section{Conclusions}

In addition to relative locomotory capacities of predator and prey, the sensory capabilities, reaction time and strategy of both participants are typically assumed to play an important role in determining the outcome of predator-prey encounters. Here we showed that the sensory capabilities, reaction time and escape strategy of prey are likely to be unimportant in situations where they are unaware of the approaching predator; rather, individual differences in routine locomotory behavior of prey strongly affected the outcome of predatory interactions in our study. Our analysis of predator-prey interactions revealed that although aerial predation in dragonflies appears to be a dynamic chase where both contestants are actively engaged in the encounter, this interaction could more accurately be described as ambush predation, as fruit flies are rarely aware of the approaching threat.

This may have broad implications for predatory behavior in dragonflies, as the likelihood of various prey types detecting their approach could play an important role in dragonflies' decisions about which prey to pursue and the best strategy to adopt. In addition, whereas most hypotheses regarding prey survival and fitness emphasize the importance of sensory capabilities and/or maximal locomotory performance during escape responses, our results suggest that routine locomotory behavior may also play a key role in determining prey fitness. More generally, the improved understanding of predator-prey interactions that results from combining ecological and mechanistic approaches may allow us to make informed predictions about the morphological, physiological and behavioral features that are likely to be under selective pressure and provide insight into the co-evolution of predator and prey.

\section{Acknowledgements}

The authors would like to thank Kelly Holt for assistance with dragonfly predation studies, Cassandra Urquhart for assistance digitizing videos and two anonymous reviewers for helpful comments on the first draft.

\section{Funding}

This work was supported by the National Science Foundation [grant 0952471 to S.A.C.].

\section{References}

Alerstam, T., Rosén, M., Bäckman, J., Ericson, P. G. P. and Hellgren, O. (2007) Flight speeds among bird species: allometric and phylogenetic effects. PLOS Biol. 5 e197.

Almbro, M. and Kullberg, C. (2008). Impaired escape ability in butterflies due to low flight muscle ratio prior to hibernation. J. Exp. Biol. 211, 24-28.

Anholt, B. R. (1991). Measuring selection on a population of damselflies with a manipulated phenotype. Evolution 45, 1091-1106.

Anholt, B. R., Marden, J. H. and Jenkins, D. M. (1991). Patterns of mass gain and sexual dimorphism in adult dragonflies (Insecta: Odonata). Can. J. Zool. 69, 11561163. 
Baird, J. M. and May, M. L. (1997). Foraging behavior of Pachydiplax longipennis (Odonoata: Libellulidae). J. Insect Behav. 10, 655-678.

Balint, C. N. and Dickinson, M. H. (2001). The correlation between wing kinematics and steering muscle activity in the blowfly Calliphora vicina. J. Exp. Biol. 204, 42134226.

Blake, R. W. and Chan, K. H. S. (2006). Models of the turning and fast-start swimming dynamics of aquatic vertebrates. J. Fish Biol. 69, 1824-1836.

Chai, P. and Millard, D. (1997). Flight and size constraints: hovering performance of large hummingbirds under maximal loading. J. Exp. Biol. 200, 2757-2763.

Combes, S. A., Crall, J. D. and Mukherjee, S. (2010). Dynamics of animal movement in an ecological context: dragonfly wing damage reduces flight performance and predation success. Biol. Lett. 6, 426-429.

Dawkins, R. and Krebs, J. R. (1979). Arms races between and within species. Proc. R. Soc. Lond. B 205, 489-511.

Dial, K. P., Greene, E. and Irschick, D. J. (2008). Allometry of behavior. Trends Ecol. Evol. 23, 394-401.

Domenici, P. (2001). The scaling of locomotor performance in predator-prey

encounters: from fish to killer whales. Comp. Biochem. Physiol. 131A, 169-182.

Domenici, P. and Blake, R. W. (1997). The kinematics and performance of fish faststart swimming. J. Exp. Biol. 200, 1165-1178.

Fry, S. N., Rohrseitz, N., Straw, A. D. and Dickinson, M. H. (2009). Visual control of flight speed in Drosophila melanogaster. J. Exp. Biol. 212, 1120-1130.

Ghose, K., Horiuchi, T. K., Krishnaprasad, P. S. and Moss, C. F. (2006) Echolocating bats use a nearly time-optimal strategy to intercept prey. PLoS Biol. 4, e108.

Ghose, K., Triblehorn, J. D., Bohn, K., Yager, D. D. and Moss, C. F. (2009).

Behavioral responses of big brown bats to dives by praying mantises. J. Exp. Biol. 212, 693-703.

Hanski, I., Henttonen, H., Korpimäki, E., Oksanen, L. and Turchin, P. (2001)

Small-rodent dynamics and predation. Ecology 82, 1505-1520.

Harper, D. G. and Blake, R. W. (1991). Prey capture and the fast-start performance of northern pike Esox lucius. J. Exp. Biol. 155, 175-192.

Hedenström, A. and Rosén, M. (2001). Predator versus prey: on aerial hunting and escape strategies in birds. Behav. Ecol. 12, 150-156.

Hedrick, T. L. (2008). Software techniques for two- and three-dimensional kinematic measurements of biological and biomimetic systems. Bioinsp. Biomim. 3, 034001.

Higham, T. E. (2007). Feeding, fins and braking maneuvers: locomotion during prey capture in centrarchid fishes. J. Exp. Biol. 210, 107-117.

Howland, H. C. (1974). Optimal strategies for predator avoidance: the relative importance of speed and manoeuvrability. J. Theor. Biol. 47, 333-350.

Jantzen, B. and Eisner, T. (2008). Hindwings are unnecessary for flight but essential for execution of normal evasive flight in Lepidoptera. Proc. Natl. Acad. Sci. USA 105 16636-16640.

Juanes, F. and Conover, D. O. (1994) Piscivory and prey size selection in young-ofthe-year bluefish: predator preference or size-dependent capture success? Mar. Ecol. Prog. Ser. 114, 59-69.

Marden, J. H. (1989). Bodybuilding dragonflies: costs and benefits of maximizing fligh muscle. Physiol. Zool. 62, 505-521.

Marden, J. H. (1994). From damselflies to pterosaurs: how burst and sustainable flight performance scale with size. Am. J. Physiol. 266, R1077-R1084.

Marden, J. H., Wolf, M. R. and Weber, K. E. (1997). Aerial performance of Drosophila melanogaster from populations selected for upwind flight ability. J. Exp. Biol. 200, 2747-2755.

Maresh, J. L., Fish, F. E., Nowacek, D. P., Nowacek, S. M. and Wells, R. S. (2004) High performance turning capabilities during foraging by bottlenose dolphins (Tursiops truncatus). Mar. Mamm. Sci. 20, 498-509.

McLachlan, A., Ladle, R. and Crompton, B. (2003). Predator-prey interactions on the wing: aerobatics and body size among dance flies and midges. Anim. Behav. 66, 911-915.
Meager, J. J., Domenici, P., Shingles, A. and Utne-Palm, A. C. (2006). Escape responses in juvenile Atlantic cod Gadus morhua L.: the effects of turbidity and predator speed. J. Exp. Biol. 209, 4174-4184.

Montooth, K. L., Marden, J. H. and Clark, A. G. (2003). Mapping determinants of variation in energy metabolism, respiration and flight in Drosophila. Genetics 165, 623-635.

Mronz, M. and Lehmann, F.-O. (2008). The free-flight response of Drosophila to motion of the visual environment. J. Exp. Biol. 211, 2026-2045.

Nyberg, D. W. (1971). Prey capture in the largemouth bass. Am. Midland Naturalist 86, 128-144.

Paine, R. T. (1966). Food web complexity and species diversity. Am. Nat. 100, 64-76.

Olberg, R. M., Worthington, A. H. and Venator, K. R. (2000). Prey pursuit and interception in dragonflies. J. Comp. Physiol. A 186, 155-162.

Olberg, R. M., Worthington, A. H., Fox, J. L., Bessette, C. E. and Loosemore, M. P. (2005). Prey size selection and distance estimation in foraging adult dragonflies. J. Comp. Physiol. A 191, 791-797.

Olberg, R. M., Seaman, R. C., Coats, M. I. and Henry, A. F. (2007). Eye movements and target fixation during dragonfly prey-interception flights. J. Comp. Physiol. A 193, 685-693.

Rand, D. M. and Lauder, G. V. (1981). Prey capture in the chain pickerel, Esox niger: correlations between feeding and locomotor behavior. Can. J. Zool. 59, 1072 1078.

Schreifer, J. E. and Hale, M. E. (2004). Strikes and startles of northern pike (Esox lucius): a comparison of muscle activity and kinematics between S-start behaviors. J. Exp. Biol. 207, 535-544.

Srygley, R. B. and Dudley, R. (1993). Correlations of the position of center of mass with butterfly escape tactics. J. Exp. Biol. 174, 155-166.

Srygley, R. B. and Kingsolver, J. G. (2000). Effects of weight loading on flight performance and survival of palatable Neotropical Anartia fatima butterflies. Biol. J. Linn. Soc. 70, 707-725.

Tammero, L. F. and Dickinson, M. H. (2002). The influence of visual landscape on the free flight behavior of the fruit fly Drosophila melanogaster. J. Exp. Biol. 205, 327-343.

Tobalske, B. W. and Dial, K. P. (2000). Effects of body size on take-off flight performance in the Phasianidae (Aves). J. Exp. Biol. 203, 3319-3332.

Vogel, S. (2008). Modes and scaling in aquatic locomotion. Int. Comp. Biol. 48, 702712

Walker, J. A., Ghalambor, C. K., Griset, O. L., McKenney, D. and Reznick, D. N. (2005). Do faster starts increase the probability of evading predators? Funct. Ecol. 19, 808-815.

Warrick, D. R. (1998). The turning- and linear-maneuvering performance of birds: the cost of efficiency for coursing insectivores. Can. J. Zool. 76, 1063-1079.

Webb, P. W. (1976). The effect of size on the fast-start performance of rainbow trout Salmo gairdneri, and a consideration of piscivorous predator-prey interactions. J. Exp. Biol. 65, 157-177.

Webb, P. W. (1984). Body and fin form and strike tactics of four teleost predators attacking fathead minnow (Pimephales promelas) prey. Can. J. Fish. Aquat. Sci. 41 157-165.

Webb, P. W. (1986). Effect of body form and response threshold on the vulnerability of four species of teleost prey attacked by largemouth bass (Micropterus salmoides). Can. J. Fish. Aquat. Sci. 43, 763-771.

Webb, P. W. and de Buffrénil, V. (1990). Locomotion in the biology of large aquatic vertebrates. Trans. Am. Fish. Soc. 119, 629-641.

Webb, P. W. and Skadsen, J. M. (1980). Strike tactics of Esox. Can. J. Zool. 58, 1462-1469.

Yager, D. D. and May, M. L. (1990). Ultrasound-triggered, flight-gated evasive maneuvers in the praying mantis Parasphendale agrionina. I. Free flight. J. Exp. Biol. 152, 17-39. 